\title{
Hinode/EIS observations of propagating low-frequency slow magnetoacoustic waves in fan-like coronal loops ${ }^{\star}$
}

\author{
T. J. Wang ${ }^{1,2}$, L. Ofman ${ }^{1,2}$, J. M. Davila ${ }^{2}$, and J. T. Mariska ${ }^{3}$ \\ 1 Department of Physics, Catholic University of America, 620 Michigan Avenue, Washington, DC 20064, USA \\ e-mail: wangtj@helio.gsfc.nasa.gov \\ 2 NASA Goddard Space Flight Center, Code 671, Greenbelt, MD 20771, USA \\ 3 Space Science Division, Naval Research Laboratory, Washington, DC 20375, USA
}

Received 19 May 2009 / Accepted 28 July 2009

\section{ABSTRACT}

\begin{abstract}
Aims. We report the first observation of multiple-periodic propagating disturbances along a fan-like coronal structure simultaneously detected in both intensity and Doppler shift in the Fe XII 195 A line with the EUV Imaging Spectrometer (EIS) onboard Hinode. A new application of coronal seismology is provided based on this observation.

Methods. We analyzed the EIS sit-and-stare mode observation of oscillations using the running difference and wavelet techniques. Results. Two harmonics with periods of 12 and $25 \mathrm{~min}$ are detected. We measured the Doppler shift amplitude of $1-2 \mathrm{~km} \mathrm{~s} \mathrm{~s}^{-1}$, the relative intensity amplitude of $3 \%-5 \%$ and the apparent propagation speed of $100-120 \mathrm{~km} \mathrm{~s}^{-1}$.

Conclusions. The amplitude relationship between intensity and Doppler shift oscillations provides convincing evidence that these propagating features are a manifestation of slow magnetoacoustic waves. Detection lengths (over which the waves are visible) of the $25 \mathrm{~min}$ wave are about 70-90 Mm, much longer than those of the $5 \mathrm{~min}$ wave previously detected by TRACE. This difference may be explained by the dependence of damping length on the wave period for thermal conduction. Based on a linear wave theory, we derive an inclination of the magnetic field to the line-of-sight about $59 \pm 8^{\circ}$, a true propagation speed of $128 \pm 25 \mathrm{~km} \mathrm{~s}^{-1}$ and a temperature of $0.7 \pm 0.3 \mathrm{MK}$ near the loop's footpoint from our measurements.
\end{abstract}

Key words. Sun: atmosphere - Sun: corona - Sun: oscillations - Sun: UV radiation - waves

\section{Introduction}

Quasi-periodic propagating intensity disturbances in fanlike large coronal loops were first observed with SOHO/EIT $195 \AA$ data (Berghmans \& Clette 1999), and then were confirmed with TRACE $171 \AA ̊$ data (e.g. De Moortel et al. 2000). These disturbances with a propagation speed on the order of $150 \mathrm{~km} \mathrm{~s}^{-1}$ have been interpreted as slow magnetoacoustic waves (Nakariakov et al. 2000). Detection of these waves is important for our understanding of the energy balance in the outer solar atmosphere and also highly valuable for coronal seismology (e.g. reviews by Nakariakov \& Verwichte 2005).

Many authors have found evidence of $3 \mathrm{~min}$ and $5 \mathrm{~min}$ oscillations propagating through the chromosphere and transition region into the lower corona (e.g. De Moortel et al. 2002; Marsh et al. 2003; Wang et al. 2009), supporting their origin in the photospheric $p$-modes by wave leakage (De Pontieu et al. 2005). In addition, there were also a few reports of similar propagating waves with long periods (10-15 $\mathrm{min})$ observed in coronal loops by EIT (Berghmans \& Clette 1999), TRACE (McIntosh et al. 2008), and STEREO/EUVI (Marsh et al. 2009), but their origin is still not clear. Long-period (10-30 $\mathrm{min})$ oscillations were also observed in polar coronal holes (e.g. Banerjee et al. 2009).

In this study, we detect two long-period (12 and $25 \mathrm{~min}$ ) harmonics in propagating slow magnetoacoustic waves in fanlike coronal loops with observations from the EUV Imaging

\footnotetext{
* Appendix is only available in electronic form at http://www . aanda. org
}

Spectrometer (EIS) onboard Hinode. The EIS instrument with high sensitivity and spectral resolution (Culhane et al. 2007) can achieve relative Doppler-shift measurements with an accuracy of less than $1 \mathrm{~km} \mathrm{~s}^{-1}$ (Mariska et al. 2008; van Doorsselaere et al. 2008; Erdélyi \& Taroyan 2008), providing us a good opportunity for detecting oscillations and waves in solar atmosphere.

\section{Observation}

EIS has both imaging and spectroscopic capabilities. Its spectroscopic mode can operate in a rastering mode or a sit-and-stare mode. In the latter case, the $1^{\prime \prime}$ or $2^{\prime \prime}$ slit is placed at a fixed location on the Sun, and repeated exposures are obtained while the Hinode spacecraft tracks the solar rotation.

The observations were obtained on 2007 February 1 in AR 10940. An EIS spectroheliogram was taken from 00:12 to $01: 22$ UT with the $1^{\prime \prime}$ slit and covering a $256^{\prime \prime} \times 256^{\prime \prime}$ region. A sit-and-stare observation within the region was taken from 01:32 to $07: 32$ UT with the $1^{\prime \prime} \times 512^{\prime \prime}$ slit and an exposure time of $60 \mathrm{~s}$. The data cover 17 spectral windows with a cadence of about $62 \mathrm{~s}$. This paper presents the result mainly for the Fe XII $\lambda 195$ line. The raw data were first processed using the standard routine eis_prep in Solar Software (SSW). The Fe XII 195.12 A line was then fitted with a single Gaussian profile, providing the total intensity, Doppler shift, and line width. The effect of a weak blended line, $195.18 \AA$ A (Young et al. 2009), is negligible since only relative Doppler shift variations are concerned in our study. 


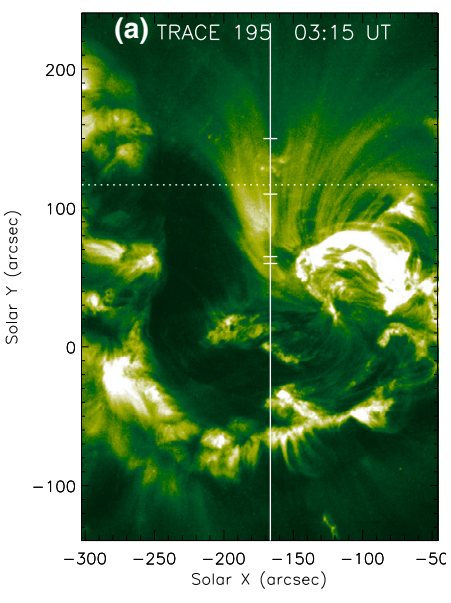

(b) EIS Fe XII 00:12 - 01:22 UT

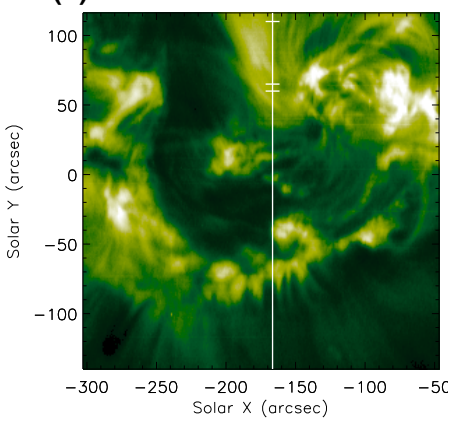

(c) Doppler Velocity

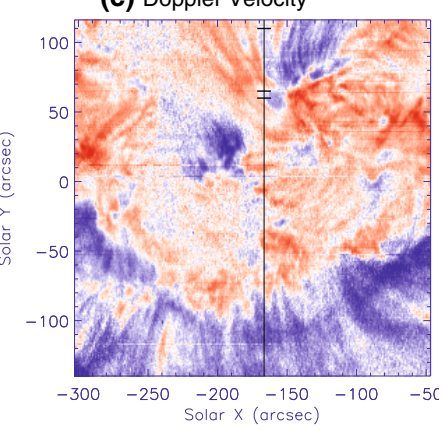

Fig. 1. a) The TRACE $\lambda 195$ bandpass image. b) The intensity map in the Fe XII $\lambda 195.12$ line from EIS. The raster observation was taken from 00:12 to 01:22 UT on 2007 February 1. c) The Doppler velocity measurements. The red color represents the redshift and the blue color the blueshift with a scale range from $-20 \mathrm{~km} \mathrm{~s}^{-1}$ to $+20 \mathrm{~km} \mathrm{~s}^{-1}$. The vertical line in each plot shows the position of the EIS $1^{\prime \prime}$ slit for the sit-and-stare observation. The short horizontal lines on the slit mark the positions where the oscillations are analyzed.
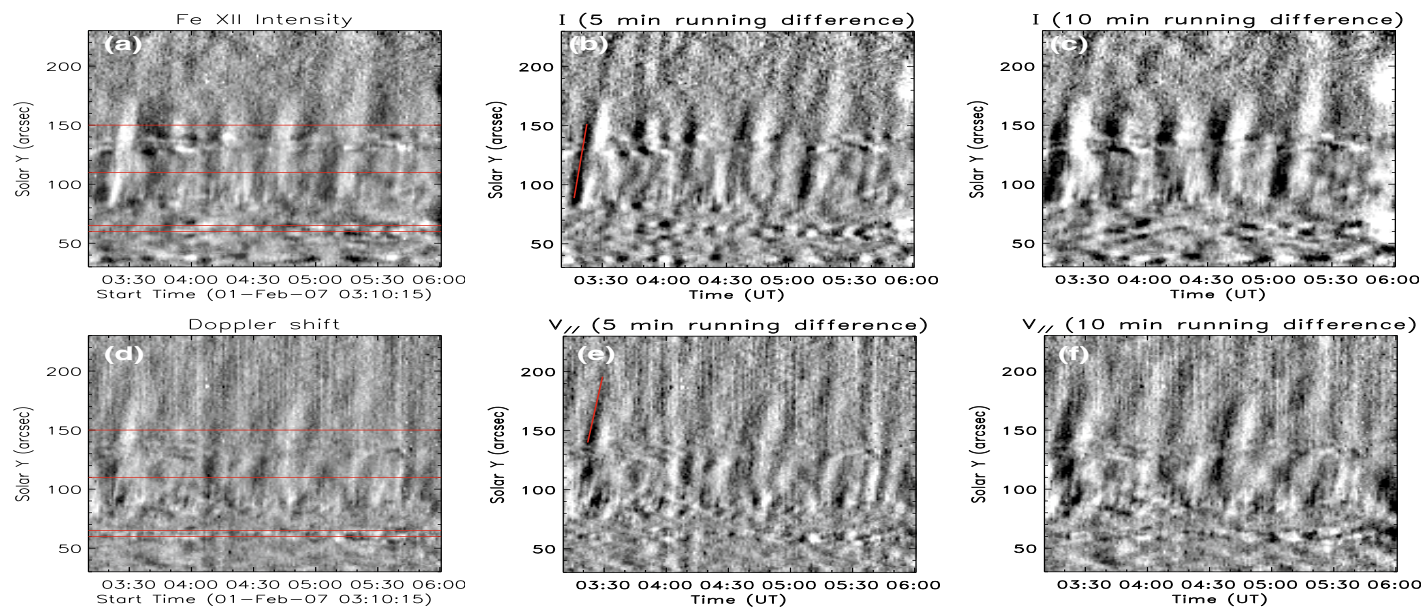

Fig. 2. The upwardly-propagating waves in coronal loops observed by Hinode/EIS. a) Time series of relative intensity along the slit in the Fe XII $\lambda 195.12$ line. b) and c) The $5 \mathrm{~min}$ and $10 \mathrm{~min}$ running difference maps for intensity. d) Time series of Doppler shift. Here the white color indicates the blueshift and the black color indicates the redshift. e) and f): the same as b) and c) but for Doppler shift. The horizontal lines in a) and d) mark three positions $\left(y=150^{\prime \prime}, 110^{\prime \prime}\right.$, and $\left.60^{\prime \prime}-65^{\prime \prime}\right)$, where the time series of oscillations are analyzed (see Figs. 3 and 4, and Fig. A.2 (online only). The lines in b) and e) outline the propagation features along the slit to demonstrate measurements of the propagation speed.

Hinode is known to have instrumental jitter in both the $x$ and $y$ directions. We estimated drifts of the pointing using the SSW routine eis_jitter from the satellite alignment data (Shimizu et al. 2007), and find that the displacements of the EIS pointing are within $3^{\prime \prime}$ during the observation. For the sit-and-stare data analyzed below, the pointing drifts in the $y$-direction have been corrected. The drifts in the $x$-direction have little effect on our result since they are slow and orbit-related with an amplitude less than $2^{\prime \prime}$ and a period of about $90 \mathrm{~min}$.

\section{Results}

A part of TRACE $195 \AA$ image recorded at 03:15 UT shows a typical fan-like set of diverging coronal loops, located at the northern edge of NOAA active region 10940 (Fig. 1a). The EIS spectroheliogram taken at 00:12 UT only covers part of this structure (Fig. 1b). The slit used for the sit-and-stare observation is almost aligned along a loop. The Doppler shift map in
EIS Fe XII $\lambda 195$ shows that the eastern part of footpoints of the fan-like loops is dominated by redshifts, while the western part of footpoints is dominated by blueshifts (Fig. 1c). The sit-andstare mode observations show weak blueshifts less than $7 \mathrm{~km} \mathrm{~s}^{-1}$ at a region of $y=90^{\prime \prime}-120^{\prime \prime}$.

Time-distance maps in intensity and Doppler shift for the EIS Fe XII line are shown in Fig. 2. To enhance weak propagating intensity disturbances, the time series of relative intensity were normalized to the background trend at each position along the slit by $\left[I\left(t, y_{i}\right)-I_{\mathrm{bg}}\left(t, y_{i}\right)\right] / I_{\mathrm{bg}}\left(t, y_{i}\right)$ (Fig. 2a), where $I_{\mathrm{bg}}\left(t, y_{i}\right)$ is the background trend at $y=y_{i}$ taken as a 20 min average smoothing of $I\left(t, y_{i}\right)$, while the $30 \mathrm{~min}$ average smoothing gives almost no difference. The background trend was also subtracted from the time series of Doppler shift with a similar method (Fig. 2d). The upwardly propagating disturbances can be seen in both intensity and Doppler shift as weak bright and dark bands with the positive gradient. The propagating disturbances are slightly affected by a small bright point at $y=130^{\prime \prime}-140^{\prime \prime}$. The footpoint of the 


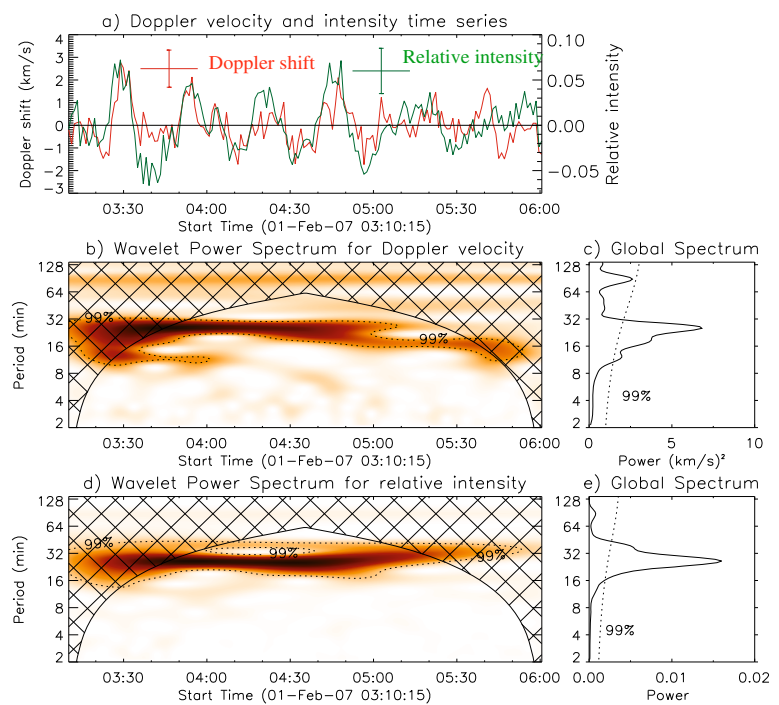

Fig. 3. Wavelet analysis for oscillation 1 at $y=150^{\prime \prime}$. a) Time profiles of Doppler shift and relative intensity. Here positive values for the Doppler shift represent the blueshifted emission. b) The wavelet power spectrum for Doppler shift. The dark color represents high power and the dotted contour encloses regions of greater than $99 \%$ confidence for a whitenoise process. c) The global wavelet spectrum (solid line) and its $99 \%$ confidence level (dotted line). d) and e): The same as b) and c) but for relative intensity.

loop (at $y=80^{\prime \prime}-100^{\prime \prime}$ ) appears to be characterized by the relatively high-frequency features, while the upper part is dominated by the relatively low-frequency features. In other words, the lower frequency component appears to propagate over a longer distance than the higher frequency one. These time-variable features can be enhanced in a running difference image, which was created by subtracting from each frame a frame taken some exposures later. The middle and right panels of Fig. 2 show the $5 \mathrm{~min}$ and $10 \mathrm{~min}$ running difference images, which clearly reveal the presence of multiple-period modes in the propagating disturbances. The propagation velocity transverse to the line of sight is estimated as the gradient of the lines outlining the evident slanting bands in the running difference images (see two examples shown in Figs. $2 b$ and $2 \mathrm{e}$ ). For nine propagation features selected for intensity and Doppler shift separately, we measured the projected propagation speed of $118 \pm 34 \mathrm{~km} \mathrm{~s}^{-1}$ for intensity and $105 \pm 25 \mathrm{~km} \mathrm{~s}^{-1}$ for Doppler shift. In addition, we also examined the coordinated TRACE data, but no corresponding propagating features are found in the constructed time-distance image along the EIS slit (see Fig. A.1 online). This may come from the lower sensitivity of TRACE compared to EIS.

The periods of the disturbances were investigated using a wavelet analysis method (Torrence \& Compo 1998). The Morlet wavelet was chosen for the convolution of time series. The intensity and Doppler shift oscillations at three positions $\left(y=150^{\prime \prime}\right.$, $110^{\prime \prime}$, and $60^{\prime \prime}-65^{\prime \prime}$, denoted as oscillations 1,2 , and 3 ) were selected for analysis. Oscillation 1 is located at the position of a loop with a projected distance of about $50 \mathrm{Mm}$ away from the footpoint, near which oscillation 2 is measured. Oscillation 3 was selected for comparison, located at another loop intersecting the slit (Fig. 1a). The time profiles used for the analysis of oscillation 3 were obtained by averaging over six pixels along the slit because the oscillation region varied slightly in the $y$-direction during the observation. In practice, we first subtracted the background trend for both intensity and Doppler shift, which was obtained by temporally smoothing the series. Since oscillation 1 is
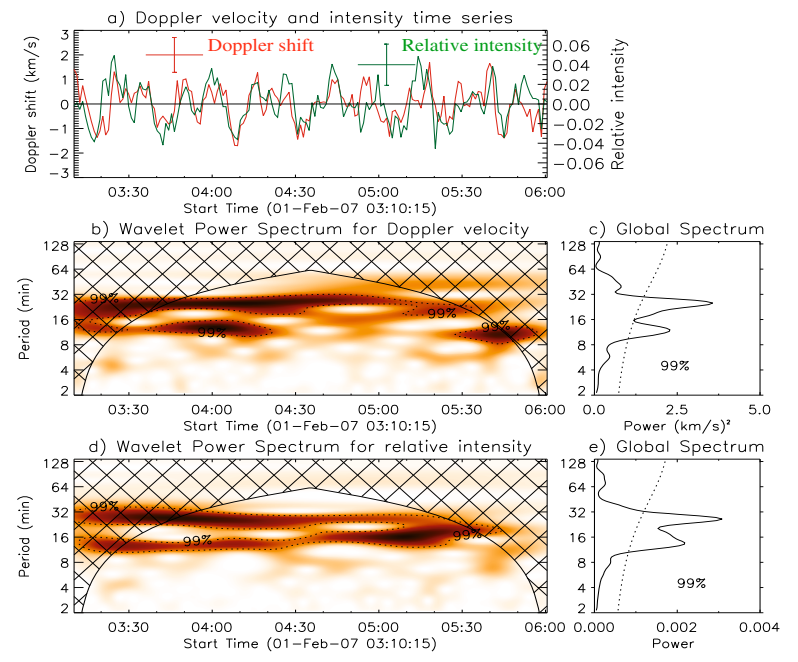

Fig. 4. Wavelet analysis for oscillation 2 at $y=110^{\prime \prime}$. The annotations are same as in Fig. 3.

dominated by the lower-frequency wave component, the background is taken as a 20 min average smoothing. To emphasize the shorter-frequency wave component in oscillations 2 and 3, the background is chosen as a 10 min average smoothing.

The evolution of detrended relative intensity and Doppler shift for three oscillations is shown in panels (a) of Figs. 3 and 4, and of Fig. A.2 (online only). There is a good in-phase relationship between the intensity and Doppler velocity, which is consistent with the signature as predicted by linear MHD wave theory for an upwardly propagating slow magnetoacoustic wave in coronal loops. The wavelet spectrum and the global wavelet spectrum are shown in panels (b)-(e) of Figs. 3 and 4, and of Fig. A.2 (online only). Cross-hatched regions in the wavelet spectrum indicate the "cone of influence", where edge effects become important due to the finite length of time series. The global wavelet spectrum is the average of the wavelet power over time at each oscillation period.

For oscillation 1 the wavelet spectra for both intensity and Doppler shift shows strong power at the period in a range of 20-30 min over a duration of 6 cycles. From the global wavelet spectra, the oscillation period for Doppler shift is measured to be $25.6 \pm 6.3 \mathrm{~min}$ and the period for intensity is $26.2 \pm 4.9 \mathrm{~min}$. The uncertainties are taken as the half FWHM of the peak. The average maximum amplitude for Doppler shift is $1.6 \pm 0.5 \mathrm{~km} \mathrm{~s}^{-1}$ and for relative intensity is $(4.9 \pm 1.5) \%$, where the errors are the standard deviation of absolute peak values. As a comparison, we also examined the case with a subtraction of the 30 min smoothing background (see Fig. A.3 online), and find that the power of the 26 min band shows almost no change, while the power of the 88 min band for Doppler shift rises above the $99 \%$ confidence level, which is caused by the orbital effect.

Oscillation 2 clearly shows that most of the wavelet power is concentrated within two period bands ranging from 8-16 min and from 20-30 min, indicating that the waves near the footpoint consist of multiple harmonic modes. The two periods are measured to be $11.9 \pm 2.8 \mathrm{~min}$ and $25.1 \pm 7.0 \mathrm{~min}$ for Doppler shift, and $13.5 \pm 4.7 \mathrm{~min}$ and $26.2 \pm 5.0 \mathrm{~min}$ for intensity. The average maximum amplitude for Doppler shift is $1.3 \pm 0.3 \mathrm{~km} \mathrm{~s}^{-1}$ and for intensity is $(3.8 \pm 0.6) \%$. Oscillation 3 shows most of the wavelet powers for Doppler shift and intensity concentrated in a range of 8-14 min. The measurements of periods and amplitudes for oscillations 1-3 are summarized in Table A.1 (online only). 
We show a new application of coronal seismology based on interpreting our observation in terms of slow magnetoacoustic waves. Assuming that the coronal field, along which the waves propagate, has an inclination, $\phi$, to the line of sight, we have

$\sin \phi=\frac{V_{\mathrm{p}}}{c_{\mathrm{s}}}$,

where $V_{\mathrm{p}}$ is the projected propagation speed of the waves and $c_{\mathrm{s}}$ the sound speed, which is a good approximation to the phase speed of slow magnetoacoustic waves under a typical coronal condition of low- $\beta$ plasma. The linearized continuity equation gives $\rho^{\prime} / \rho_{0}=V^{\prime} / c_{\mathrm{s}}$, where $\rho_{0}$ is the undisturbed loop density, and $\rho^{\prime}$ and $V^{\prime}$ the density and velocity perturbations, respectively. Considering $I^{\prime} / I_{0} \sim 2 \rho^{\prime} / \rho_{0}$ and $\cos \phi=V_{\|} / V^{\prime}$, where $I^{\prime} / I_{0}$ is the relative amplitude for intensity disturbances and $V_{\|}$ the Doppler shift amplitude, we obtain

$\cos \phi=\frac{2 V_{\|}}{\left(I^{\prime} / I_{0}\right) c_{\mathrm{s}}}$.

From Eqs. (1) and (2) we derive the inclination angle,

$\phi=\tan ^{-1}\left[\frac{1}{2} V_{\mathrm{p}}\left(\frac{I^{\prime} / I_{0}}{V_{\|}}\right)\right]$.

Note that Eq. (3) also holds in the condition with a steady flow in the loop. We take $V_{\mathrm{p}}=110 \pm 30 \mathrm{~km} \mathrm{~s}^{-1}$, the mean of the projected propagation speeds measured for intensity and Doppler shift. By assuming that the inclination of the coronal field is constant over the detection length and taking the quantity of $\left(I^{\prime} / I_{0}\right) / V_{\|}$to be the mean value of the measurements for oscillations 1 and 2, we obtain $\phi=59^{\circ} \pm 8^{\circ}$ from Eq. (3). Then with Eq. (1) the sound speed is estimated to be $128 \pm 25 \mathrm{~km} \mathrm{~s}^{-1}$. From $c_{\mathrm{s}}=0.152 T^{1 / 2}$ $\mathrm{km} \mathrm{s}^{-1}$ we estimate the average temperature of the loop near the footpoint about $0.7 \pm 0.3 \mathrm{MK}$. This result is in good agreement with what is obtained from observations of 3D propagation of similar waves with STEREO/EUVI (Marsh et al. 2009).

\section{Discussion}

We have reported on the first observation of the multiple-period (12 and $25 \mathrm{~min}$ ) propagating disturbances along a fan-like coronal structure in both intensity and Doppler shift in the Fe XII $195 \AA$ line with Hinode/EIS. The phase and amplitude relationships between the intensity and Doppler shift oscillations provide convincing evidence that they are a propagating slow magnetoacoustic wave.

With high cadence (15 s) EIT $195 \AA$ observations, Berghmans \& Clette (1999) first detected the propagating intensity disturbances in fanlike coronal loops. They noticed that these disturbances are associated with recurrent footpoint brightenings, appearing as transient flows ejected periodically. In our case the propagating features are detected in both intensity and Doppler shift. The small Doppler shift amplitude of several $\mathrm{km} \mathrm{s}^{-1}$ is consistent with the intensity amplitude as expected for a slow magnetoacoustic wave, thus, interpreting these oscillations in terms of propagating slow magnetosonic waves is more appropriate. In addition, the apparent propagation speed of the oscillations $>100 \mathrm{~km} \mathrm{~s}^{-1}$ is an order of magnitude higher than any flow speeds detected in this region.

In our case the dominant oscillations have periods of about 25 min, much longer than those observed in the TRACE loops of around 5 min (De Moortel et al. 2002). It is not clear why the longer periods reported here were not observed in TRACE loops. One reason for De Moortel et al. (2002) detecting only the 5 min oscillations could be attributed to the selection criterion they used, where a running difference technique with time lag of $90 \mathrm{~s}$ would eliminate the long-period components that may exist in some cases. De Pontieu et al. (2005) suggest that 5 min waves in coronal loops come from the leakage of $p$-modes through the chromosphere tunneled by highly inclined magnetic fields. However, the origin of 20-30 min waves is difficult to explain by such a mechanism because their period is much longer than the cutoff period of the slow magnetoacoustic waves in the chromosphere (Bel \& Leroy 1977). Therefore, another explanation is required, e.g., possibly the coupling with the photospheric $f$-mode caused by local convective motions.

The detection length is defined as the distance along the loop over which the intensity disturbances can be observed. TRACE observations show that the waves are damped quickly with a detection length typically in the range of 3-23 Mm (De Moortel et al. 2002). Theoretical and numerical studies show that thermal conduction can play an important role in the quick damping of slow magnetosonic waves in coronal loops (e.g. Ofman \& Wang 2002; De Moortel \& Hood 2004; Klimchuk et al. 2004). In our case the detection length is measured to be about $69 \mathrm{Mm}$ for intensity and about $87 \mathrm{Mm}$ for Doppler velocity, much longer than in the TRACE case. This difference may be explained by the dependence of the damping length on the wavelength and the temperature of the ambient plasma (Klimchuk et al. 2004). Thus, the longer period waves reported here have longer wavelength than in previous observations, resulting in weaker damping by thermal conduction. In addition, the present loop appears cooler ( $~ 0.7 \mathrm{MK}$ as estimated in the previous section) than previously observed loops (1.0-1.5 MK for TRACE loops).

Acknowledgements. Hinode is a Japanese mission developed and launched by ISAS/JAXA in partnership with NAOJ, NASA, and STFC (UK). Additional operation support is provided by ESA and NSC (Norway). The authors are grateful to Dr. Harry Warren for his planning of EIS observations. The work of L.O. and T.J.W. was supported by NRL grant N00173-06-1-G033. L.O. was also supported by NASA grant NNG06GI55G. The authors also thank the referee, Dr. Dipankar Banerjee, for his constructive comments and suggestions.

\section{References}

Banerjee, D., Teriaca, L., Gupta, G. R., et al. 2009, A\&A, 499, L29 Bel, N., \& Leroy, B. 1977, A\&A, 55, 239

Berghmans, D., \& Clette, F. 1999, Sol. Phys., 186, 207

Culhane, J. L., Harra, L. K., James, A. M., et al. 2007, Sol. Phys., 243, 19 De Moortel, I., \& Hood, A. W. 2004, A\&A, 415, 705

De Moortel, I., Ireland, J., \& Walsh, R. W. 2000, A\&A, 355, L23

De Moortel, I., Ireland, J., Walsh, R. W., et al. 2002, Sol. Phys., 209, 61

De Pontieu, B., Erdélyi, R., \& De Moortel, I. 2005, ApJ, 624, L61

Erdélyi, R., \& Taroyan, Y. 2008, A\&A, 489, L49

Klimchuk, J. A., Tanner, S. E. M., \& De Moortel, I. 2004, ApJ, 616, 1232

Mariska, J. T., Warren, H. P., Ugarte-Urra, I., et al. 2008, PASJ, 59, S713

Marsh, M. S., Walsh, R. W., De Moortel, I., et al. 2003, A\&A, 404, L37

Marsh, M. S., Walsh, R. W., \& Plunkett, S. 2009, ApJ, 697, 1674

McIntosh, S. W., De Pontieu, B., \& Tomczyk, S. 2008, Sol. Phys., 252, 321

Nakariakov, V. M., \& Verwichte, E. 2005, Living Reviews in Solar Physics, 2, 3 , http: //www . livingreviews . org/lrsp-2005-3

Nakariakov, V. M., Verwichte, E., Berghmans, D., et al. 2000, A\&A, 362, 1151

Ofman, L., \& Wang, T. J. 2002, ApJ, 580, L85

Shimizu, T., Katsukawa, Y., Matsuzaki, K., et al. 2007, PASJ, 59, 845

Torrence, C., \& Compo, G. P. 1998, Bull. Meteor. Soc., 79, 61

van Doorsselaere, T., Nakariakov, V. M., Young, P. R., et al. 2008, A\&A, 487, L17

Wang, T. J., Ofman, L., \& Davila, J. M. 2009, ApJ, 696, 1448

Young, P. R., Watanabe, T., Hara, H., et al. 2009, A\&A, 495, 587 
T. J. Wang et al.: Propagating low-frequency slow magnetoacoustic waves in coronal loops, Online Material p 1

\section{Appendix A: Online materials}
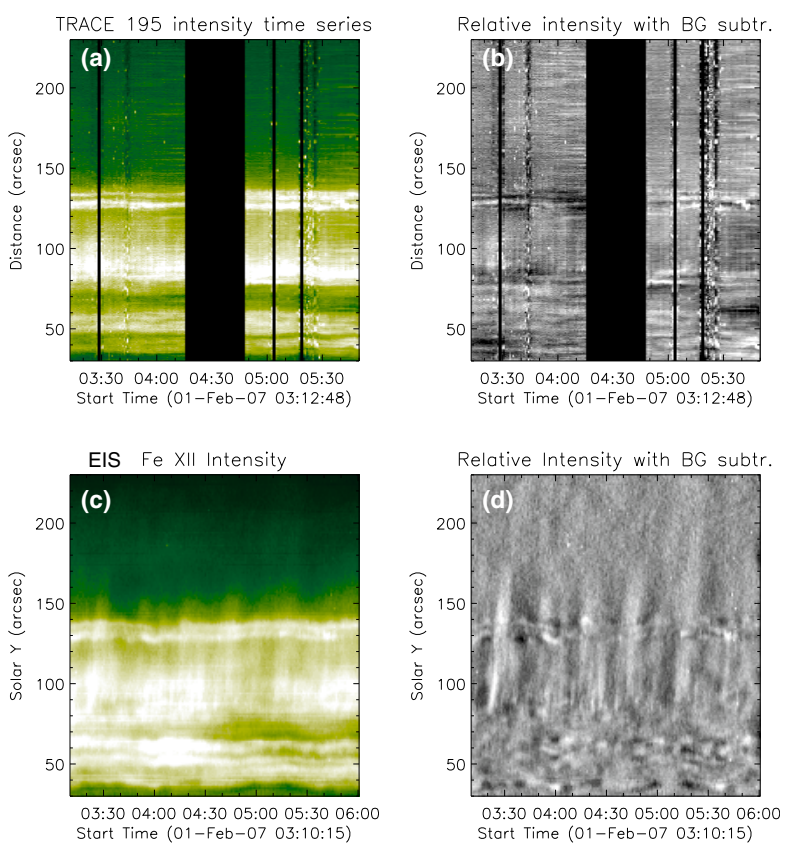

Fig. A.1. Comparisons between EIS and TRACE observations. a) Constructed TRACE $195 \AA$ time-distance image along the EIS slit. b) Same as a) but with the background subtraction, where the background is taken as the average over time at each position along the cut. c) The EIS Fe XII intensity time series. d) Same as c) but with the background subtraction.
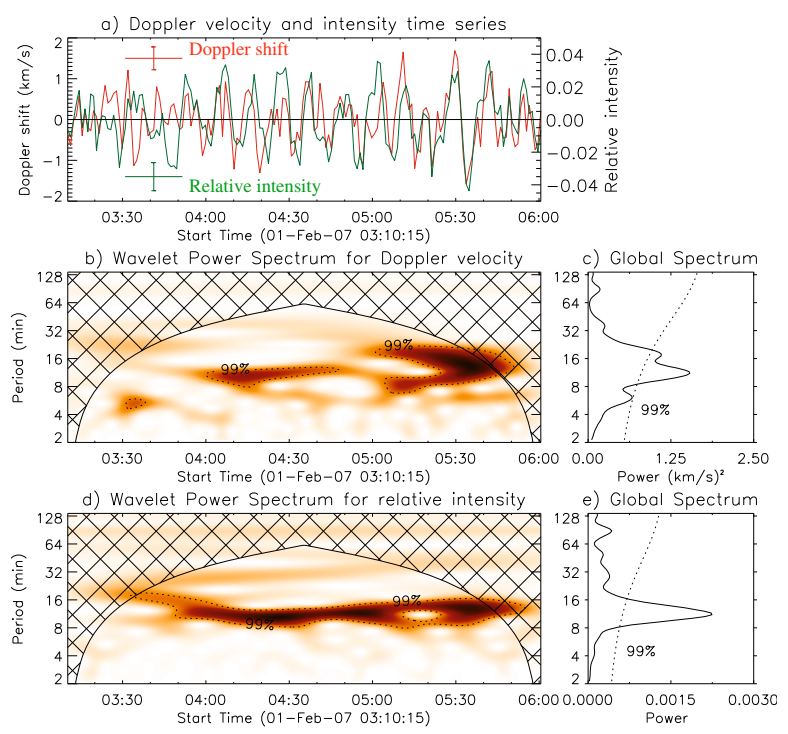

Fig. A.2. Wavelet analysis for oscillation 3 averaged over $y=60^{\prime \prime}-65^{\prime \prime}$. The annotations are same as in Fig. 3.
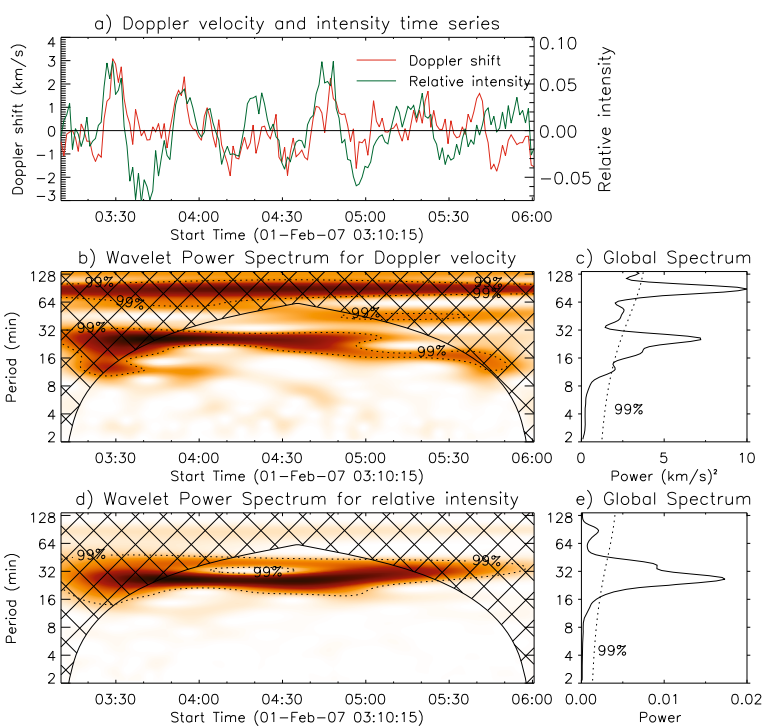

Fig. A.3. Wavelet analysis for oscillation 1 at $y=150^{\prime \prime}$, which is the same as Fig. 3 but with a subtraction of the background taken as a 30 min average smoothing.

Table A.1. Measurements of the oscillations in fanlike loops found in the EIS sit-and-stare data in the Fe XII (195 ̊) line $^{a}$.

\begin{tabular}{lcccc}
\hline \hline $\begin{array}{l}\text { Oscillation } \\
\text { No. }\end{array}$ & $\begin{array}{c}P_{V} \\
(\mathrm{~min})\end{array}$ & $\begin{array}{c}P_{I} \\
(\mathrm{~min})\end{array}$ & $\begin{array}{c}A_{V} \\
\left(\mathrm{~km} \mathrm{~s}^{-1}\right)\end{array}$ & $\begin{array}{c}A_{I} \\
(\%)\end{array}$ \\
\hline 1 & $25.6 \pm 6.3$ & $26.2 \pm 4.9$ & $1.6 \pm 0.5$ & $4.9 \pm 1.5$ \\
$2^{b}$ & $25.1 \pm 7.0$ & $26.2 \pm 5.0$ & $1.3 \pm 0.3$ & $3.8 \pm 0.6$ \\
& $11.9 \pm 2.8$ & $13.5 \pm 4.7$ & - & - \\
3 & $11.2 \pm 3.8$ & $11.2 \pm 2.9$ & $1.0 \pm 0.3$ & $2.8 \pm 0.7$ \\
\hline
\end{tabular}

${ }^{a} P_{V}$ and $P_{I}$ are periods and $A_{V}$ and $A_{I}$ are amplitudes for the Doppler shift and relative intensity oscillations, respectively; ${ }^{b}$ two harmonic components are found for oscillation 2. 